\title{
Search for PeV Gamma-Ray Point Sources with IceCube
}

\section{The IceCube Collaboration ${ }^{\dagger}$}

$\dagger$ http://icecube.wisc.edu/collaboration/authors/icrc17_icecube

E-mail: zachary.griffith@icecube.wisc.edu

\begin{abstract}
We present results of a search for PeV gamma-ray point sources with the IceCube Neutrino Observatory, presently the most sensitive facility for $\mathrm{PeV}$ gamma-ray sources in the southern hemisphere. This includes a general search over IceCube's field of view, as well as tests for correlations with $\mathrm{TeV}$ sources detected by H.E.S.S. and neutrino events from IceCube's high-energy starting event sample. As the attenuation length of PeV gamma rays limits observational distances to within the Galaxy, one of the goals of the analysis is to constrain the Galactic component of the astrophysical neutrino flux observed by IceCube.

Corresponding authors: Zachary Griffith ${ }^{* 1}$, Hershal Pandya ${ }^{2}$

${ }^{1}$ Wisconsin IceCube Particle Astrophysics Center (WIPAC) and Department of Physics, University of Wisconsin-Madison, Madison, WI 53706, USA

2 Dept. of Physics and Astronomy, University of Delaware, Newark, DE 19711, USA.
\end{abstract}

35th International Cosmic Ray Conference - ICRC2017

10-20 July, 2017

Bexco, Busan, Korea

${ }^{*}$ Speaker. 


\section{Introduction}

IceCube previously set the most stringent limit on $\mathrm{PeV}$ gamma-ray emission in the southern hemisphere at $(\mathrm{E} / \mathrm{TeV})^{2} \mathrm{~d} \Phi / \mathrm{dE} \approx 10^{-18}-10^{-17} \mathrm{~cm}^{-2} \mathrm{~s}^{-1} \mathrm{TeV}^{-1}$ using one year of data taken with a partial detector during construction [1]. We present here an updated $\mathrm{PeV}$ gamma-ray analysis with IceCube using five years of data and an enhanced event selection. We use this analysis to perform an all-sky scan for point sources of $\mathrm{PeV}$ gamma rays as well as correlation tests with known Galactic TeV gamma-ray sources and IceCube's high-energy starting event (HESE) sample of neutrinos.

All known Galactic TeV gamma-ray sources in IceCube's field of view have been detected by the High Energy Stereoscopic System (H.E.S.S.) [2]. Of these sources, 15 have steady emission with no evidence of a spectral break or cutoff [3]. Of those with extended emission, none approach the median angular resolution of this analysis of $\sim 0.5^{\circ}$ and the sources are treated as point-like. Each source is treated individually in addition to a stacked catalog. This analysis is the first to test for a signal from these sources at higher energies, and an extension up to $\mathrm{PeV}$ energies from many of these sources can be constrained by this analysis.

If the HESE neutrinos include a galactic component, an associated $\mathrm{PeV}$ gamma-ray flux has been predicted by several studies ([4], [5]). This is due to the fact that the same hadronic interactions that produce $\mathrm{PeV}$ neutrinos most likely also produce gamma rays from the decay of neutral pions. However, PeV gamma rays can only be observed over Galactic distances due to pair-production with the cosmic microwave background (CMB) radiation field [6]. Therefore, a measurement or limit on the flux of gamma rays at PeV energies constrains the Galactic component of the neutrino flux observed by IceCube.

\section{Dataset}

The IceCube Neutrino Observatory, located at the geographic South Pole, is comprised of two components. The cubic-kilometer neutrino detector IceCube [7] is installed in the ice between depths of $1450 \mathrm{~m}$ and $2450 \mathrm{~m}$. Its companion surface air shower array, IceTop [8], is located on top of the ice sheet, corresponding to an altitude of 2835 meters above sea level. Both components were completed in 2011. This analysis uses IceTop data for event energy and direction, while both IceTop and IceCube information is used in the classification of gamma rays. The greatest difference between gamma-ray and hadronic air showers that IceCube is capable of exploiting is the number of muons present in the air shower. Gamma-ray air showers do have muons from the decay of pions and kaons due to photoproduction processes in addition to muon pair production [9]. However, these processes are three orders of magnitude more rare, per shower, than the nucleusnucleus interactions which produce muons in hadronic showers [10].

To ensure good data quality (energy determination, angular resolution), the following cuts were applied. Good fits to the shower lateral distribution and core location are required for each event, and events are restricted to have passed inside the IceTop area. A zenith-dependent minimum energy cut of $\sim 0.6-0.7 \mathrm{PeV}$ is applied to ensure adequate trigger efficiency in IceTop, while a maximum energy cut of $100 \mathrm{PeV}$ is set above which no Monte Carlo was simulated and where there are few events in data. A maximum zenith angle cut of $\sim 37^{\circ}$ is also applied. 
After quality cuts, an additional event selection is applied to create an event sample rich in gamma rays. Features are selected in IceTop and IceCube that are correlated with muon content and shower age. These features are used in random forest classification to create the final event sample. The classification is implemented using the open source python software Scikit-learn [11].

IceCube is a muon detector, which means the total number of photoelectrons recorded in IceCube's photomultipliers is proportional to the high-energy $(>276 \mathrm{GeV})$ muons in the air shower for events coincident in IceTop and IceCube. Rather than a veto of all showers which have signal in IceCube as was done in Ref. [1], the number of photoelectrons is used as one parameter in a random forest classification, along with a parameter that describes the containment of the shower axis within IceCube.

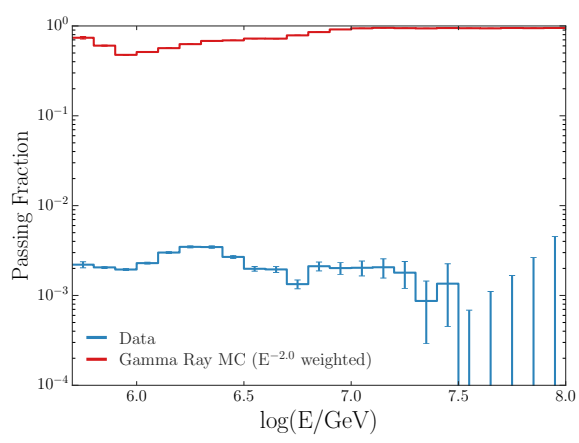

Figure 1: The fraction of events which pass the random forest classification cut from the quality cut level event sample for a section of data from the 2012 observing year and gamma-ray simulation weighted to an $E^{-2.0}$ spectrum and detector response using October 2012 snow heights.
Lower-energy muons that are laterally separate from the shower core produce a charge signature in IceTop tanks that is prominent for muon-rich cosmic-ray air showers. In addition, gamma-ray air showers interact deeper in the atmosphere than hadronic air showers, resulting in a difference in shower front curvature. The following is a brief description of the parameter developed to encapsulate this information.

Three sets of likelihood tables are constructed using information from hits in IceTop tanks: charge against distance from the shower core, hit time against distance from the shower core, and charge against time. Hadronic likelihood tables are built from data, rather than Monte Carlo simulation of cosmic rays, in order to abstain from making any composition assumption. Existing limits place the expected fraction of gamma rays to be less than $10^{-4}$ of the total cosmic ray flux [12], so the influence of signal events in the data is small.

Gamma-ray tables are built from Monte Carlo simulation of gamma rays. During event selection, the tank information of each event is used to sample from the likelihood tables and calculate a total likelihood from the hadronic shower hypothesis as well as the gamma-ray hypothesis. The parameter used for classification is the ratio of these total likelihoods, such that higher values indicate a more hadron-like shower. A full description of this technique is documented in Ref. [13]. Utilizing IceTop for event selection allows for the inclusion of events that do not pass through IceCube, which significantly boosts the acceptance to gamma rays for inclined showers.

The random forests are trained using experimental data as the background and Monte Carlo simulation of gamma rays as the signal. Two random forests were trained for this analysis: one using gamma-ray simulation weighted to an $E^{-2.0}$ spectrum and one with simulation weighted to $E^{-2.7}$. These comprise reasonable bounds for an observable point source in the dataset. The final event sample consists of data events with a random forest score of greater than 0.7 in either of the classifiers. The fraction of data and gamma-ray simulation that survive from the quality cut event samples after applying the random forest cut is shown in Fig. 1.

Five years of data from the completed detector are included in the dataset, from May 2011 to 
May 2016. Snow has continuously accumulated on the IceTop tanks over this time, which attenuates the electromagnetic component of air showers, significantly decreasing the effective area to gamma rays. Snow measurements are performed twice per year to account for the effect on IceTop data. In order to account for these variations over time, the detector response to simulated gammarays was repeated for each year of data, with the fall measurement of snow used as the simulated IceTop snow heights. Classifiers are trained for each year of data and dedicated simulation independently. The complete dataset at the final cut level is comprised of a total livetime of 1,576 days and 471,461 events.

\section{Search Methods}

The following section describes the statistical methods and three different source hypotheses tested in this analysis. We use a maximum likelihood test with the following likelihood function

$$
L=\prod_{i} \prod_{j \in i}\left(\frac{n_{s}^{j}}{N} S\left(\left|\mathbf{x}_{j}-\mathbf{x}_{S}\right|, E_{j}, \sigma_{j} ; \gamma\right)+\left(1-\frac{n_{s}^{j}}{N}\right) B\left(\delta_{j}, E_{j}\right)\right) .
$$

This likelihood $L$ is a product over $j$ events in each of i datasets, where each dataset is comprised of one year of data. Each event has a direction $\mathbf{x}_{j}$ (including declination $\delta_{j}$ ), energy $E_{j}$, and angular uncertainty $\sigma_{j}$. The events are compared to a point-source hypothesis comprised of a direction $\mathbf{x}_{S}$ and a spectral index $\gamma$. The terms $S$ and $B$ are the signal and background probability distribution functions (PDFs), respectively. For the all-sky scan they are defined as:

$$
S_{i}=\frac{1}{2 \pi \sigma_{i}^{2}} \mathrm{e}^{-\frac{\left|\mathbf{x}_{j}-\mathbf{x}_{S}\right|^{2}}{2 \sigma_{i}^{2}}} \mathscr{E}_{S, i}\left(E_{i}, \delta_{i}, \gamma\right) \quad \text { and } \quad B_{i}=\frac{1}{2 \pi} B_{\exp }\left(\delta_{i}\right) \mathscr{E}_{B, i}\left(E_{i}, \delta_{i}\right)
$$

where a Gaussian uncertainty is assumed for the signal PDF, $B_{\exp }$ is the spatial acceptance to cosmic rays derived from data, and $\mathscr{E}_{S, i}, \mathscr{E}_{B, i}$ are the normalized energy distributions for signal and background, respectively. The background PDF is uniform in right ascension and constructed from data.

To test for a correlation with H.E.S.S. sources in the analysis field of view, the signal PDF is modified to include $M$ source locations each with a Gaussian uncertainty that is event dependent:

$$
S_{i}=\frac{\sum_{m}^{M} \frac{R\left(\delta_{m}\right)}{2 \pi \sigma_{i}^{2}} \mathrm{e}^{-\frac{\left|\mathrm{x}_{j}-\mathrm{x}_{S}\right|^{2}}{2 \sigma_{i}^{2}}}}{\sum_{m}^{M} R\left(\delta_{m}\right)},
$$

where $R_{m}$ is the relative detector acceptance to gamma rays at the location of the source $m$.

To test for a correlation with IceCube HESE sample of neutrinos, the event sample is split into "cascade"-like events, which have relatively poor angular resolution $\left(\sim 8^{\circ}-10^{\circ}\right)$, and "track"like events with angular resolution $<1.2^{\circ}$. There are a total of 11 events in the 4-year HESE sample [14] with a reconstructed direction (within $1 \sigma$ uncertainty) within the field of view of this analysis. Of those, 10 are cascade events, with a single "track"-like event at a declination of $\delta=$ $-86.77^{\circ}$. The single track event is treated as a source with the signal PDF reformulated to be: 


$$
S_{i}=\frac{1}{2 \pi \sigma_{i, S}^{2}} \mathrm{e}^{-\frac{\left|\mathbf{x}_{j}-\mathbf{x}_{S}\right|^{2}}{2 \sigma_{i, S}^{2}}} \mathscr{E}_{S, i}\left(E_{i}, \delta_{i}, \gamma\right) \quad \text { and } \quad \sigma_{i, S}=\sqrt{\sigma_{i}^{2}+\sigma_{S}^{2}},
$$

where $\sigma_{S}$ is the angular uncertainty of the track event.

As the cascade events have large angular uncertainties, to properly account for the change in acceptance over the field of view a template likelihood method is used. The template is constructed by defining each cascade event to have a signal PDF as Eq. 3.4. These signal PDFs are combined and renormalized to form a signal template, which is then convolved with the detector acceptance to gamma rays. A full description of the template likelihood method is included in [15].

In all cases, the likelihood is maximized in $n_{s}$ and $\gamma$, and the ratio of the optimal likelihood to the null hypothesis $\left(n_{s}=0\right)$ yields the test statistic. To model the expected output of backgroundonly events this process is repeated many times, with each trial using a random (scrambled) set of right ascension values. The p-value of an observed test statistic is then obtained from a comparison to the ensemble of test statistic values returned by the background trials.

\section{Results}

\subsection{All-Sky Point Source Search}

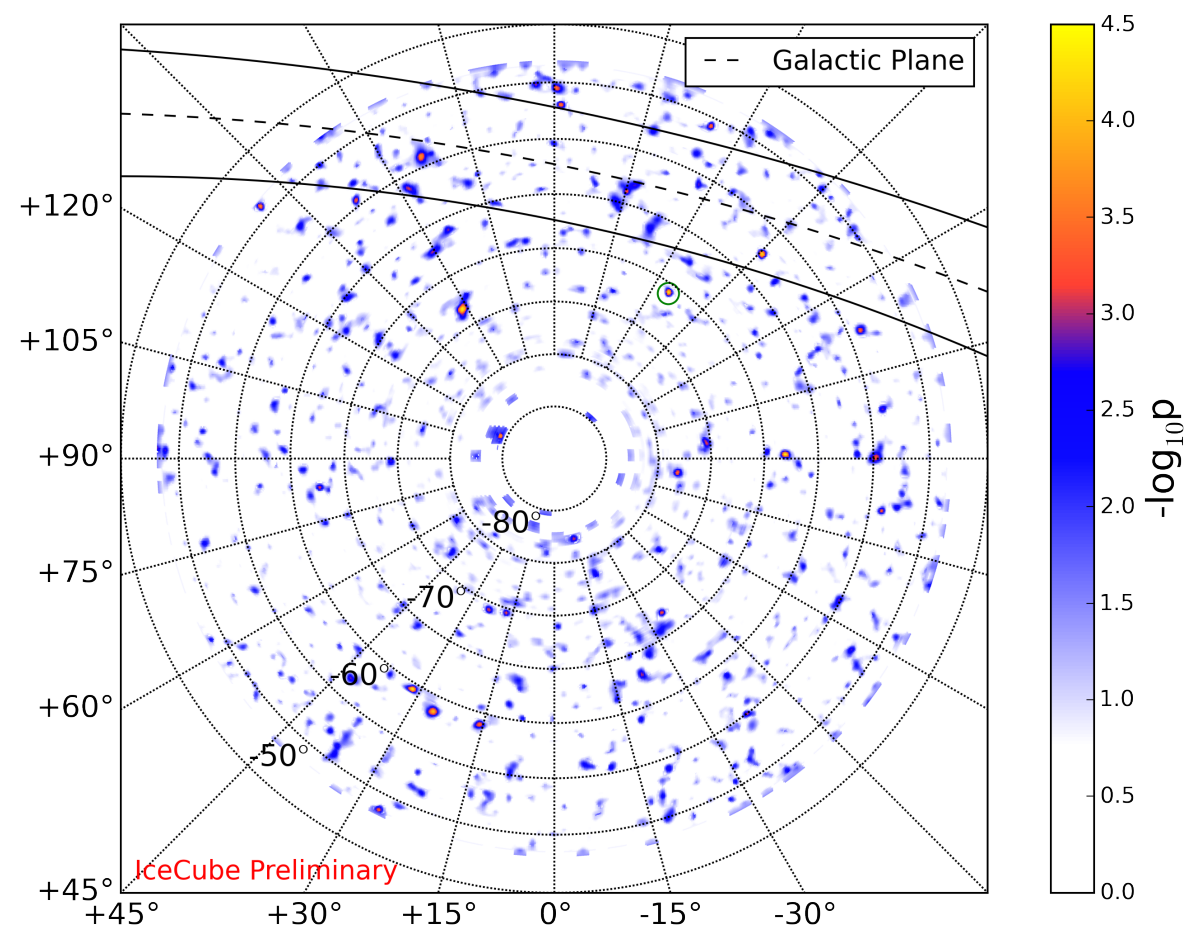

Figure 2: An equatorial polar map of the all-sky scan pre-trial p-values. The solid and dashed black line represents the Galactic plane region $\pm 10^{\circ}$. The location of the lowest p-value is circled in green.

The all sky search is accomplished by a scan over each pixel in a HEALPIX [16] map (nside=512) with a pixel diameter of $\sim 0.11^{\circ}$. For each pixel, the test statistic is evaluated given a source hy- 
pothesis at the position of the pixel. Fig. 2 shows the results of the all-sky point source search over the entire field of view, excluding the region within $5^{\circ}$ of the pole where scrambling in right ascension is insufficient to build independent background trials. The color scale corresponds to a pre-trial p-value that for each position is determined through a comparison of the test statistic value of the true event locations to that of an ensemble of background trials, each with event locations scrambled in right ascension.
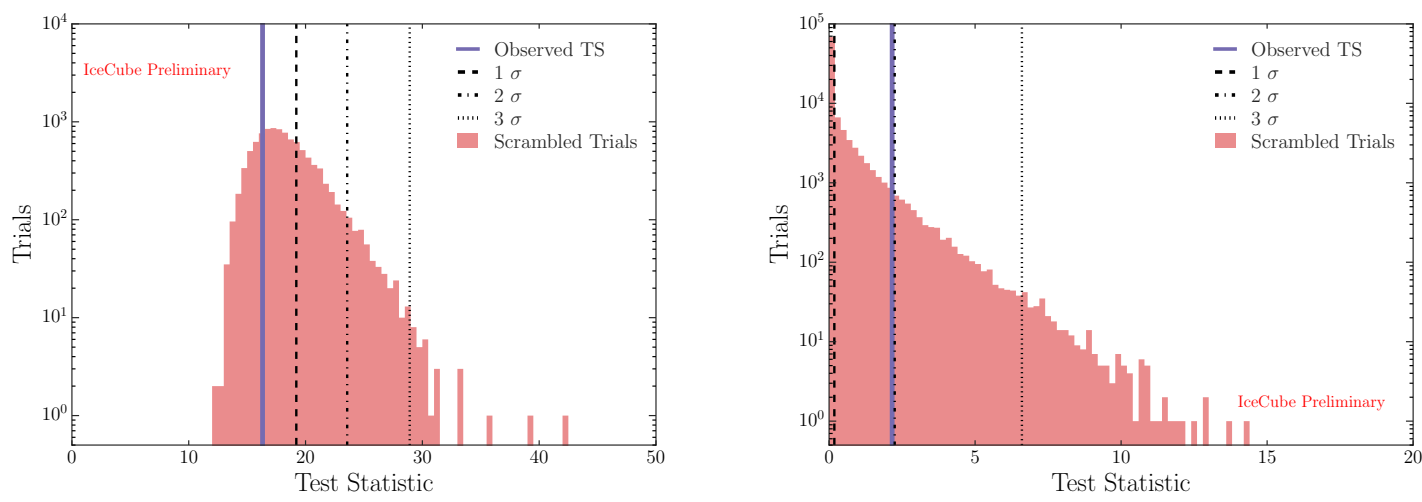

Figure 3: Left: the highest test statistic found in data compared to an ensemble of background scrambled trials. Right: result of a stacking analysis on the H.E.S.S. source catalog.

The cluster in the sky with the lowest $\mathrm{p}$-value is at $-70.9^{\circ}$ declination and $214.7^{\circ}$ right ascension, with $n_{s}=24.03$, a spectral index of 1.87, and a test statistic of 16.32. Fig. 3 (left) shows this test statistic compared to a distribution of the highest test statistic in trials of right ascension scrambled event samples, which gives a post-trial p-value of $77.4 \%$, compatible with background expectation.
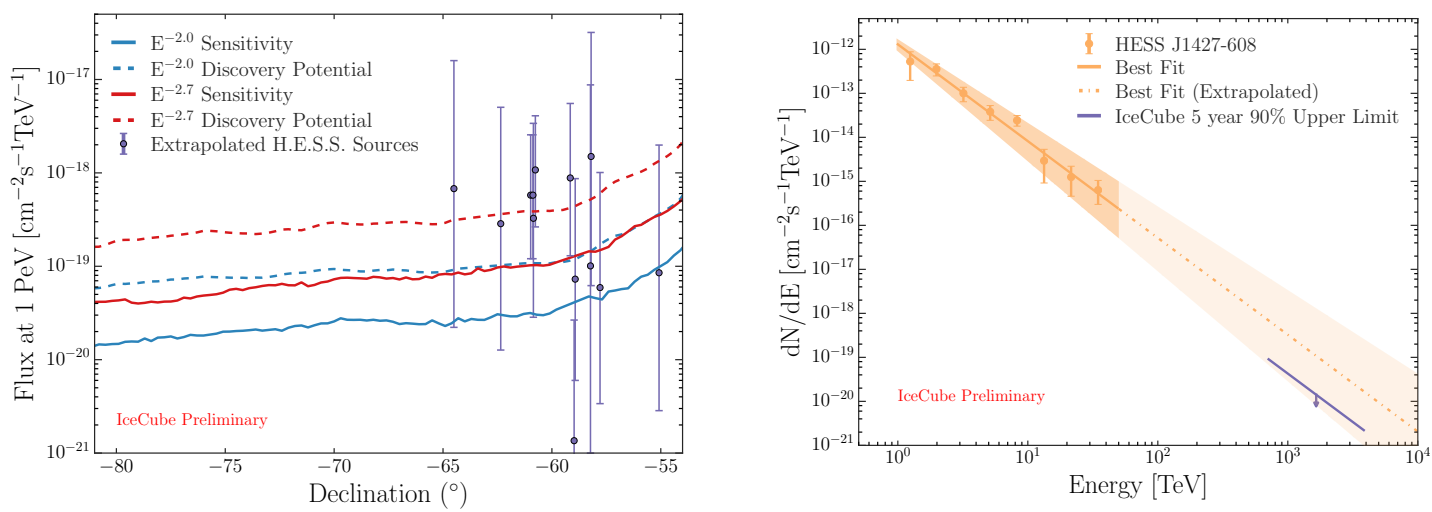

Figure 4: Left: sensitivity and discovery potential to $E^{-2.0}$ and $E^{-2.7}$ sources at $1 \mathrm{PeV}$. Shown in purple are the extrapolation up to $1 \mathrm{PeV}$ of H.E.S.S. sources in the analysis field of view under an optimistic scenario of no energy spectrum breaks and zero absorption. Right: the measured and best fit flux of H.E.S.S. J1427608 [17] along with the $90 \%$ upper limit set by this analysis.

\subsection{H.E.S.S. Sources}

Fig. 4 (left) shows the integral flux sensitivity and discovery potential as a function of decli- 
nation for the full five-year sample, along with the extrapolated flux of H.E.S.S. sources assuming no break in the energy spectrum and no absorption from radiation fields. Recent modeling has shown Galactic sources can be significantly attenuated depending on the distance from Earth and line of sight proximity to the Galactic center [18]. The points therefore represent an optimistic flux prediction for each source. Not present on the plot are two sources, SNR G292.2-00.5 and SNR G318.2+00.1, which have no reported flux values. The sensitivity of this analysis is defined as the signal flux at which $90 \%$ of experiments yield a test statistic at or above the median of an ensemble of background-only trials. This is equivalent to the Neyman construction of $90 \%$ confidence level upper limits. The discovery potential is reached with a signal flux that achieves a $5 \sigma$ post-trial p-value in $50 \%$ of experiments.

A particularly interesting source in the analysis field of view is H.E.S.S. J1427-608, which remains of an unidentified class. Guo et al. [19] reported on a counterpart seen in Fermi-LAT data at $\mathrm{GeV}$ energies with a best fit including H.E.S.S. data of $E^{-2}$ over four orders of magnitude in energy with no break in the spectrum, a property unique among currently known $\mathrm{TeV}$ sources. The extrapolated, non-attenuated flux of the source H.E.S.S. J1427-608 is shown in Fig. 4 (right) as a function of energy along with the $90 \%$ integral flux upper limit set by this analysis, for which the energy bounds are the 5\% and $95 \%$ quantiles of the data energy distribution. The upper limit set by this analysis rejects this optimistic scenario and can help constrain models of the source class.

Fig. 5 shows the H.E.S.S. sources included in this analysis, which were selected from the TeVCat catalog. The source with the lowest pre-trial p-value, H.E.S.S. J1026-582, has a post-trial p-value of $44.7 \%$. A test statistic of 2.16 is returned by the stacking analysis, which is shown compared to the background trial test statistic distribution in Fig. 3 (right). The resulting p-value is $5.4 \%$.

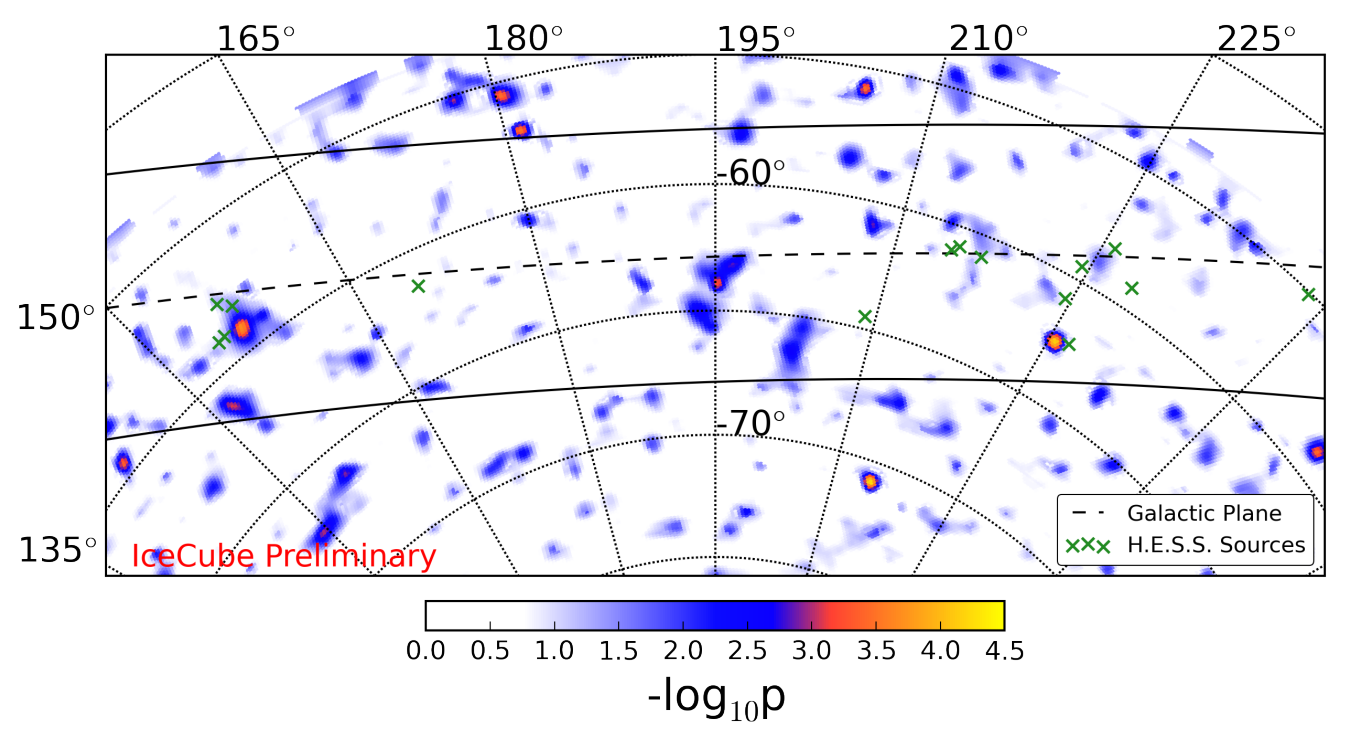

Figure 5: Map of p values from the all-sky scan with H.E.S.S. source directions overlaid. 


\subsection{Correlation with IceCube HESE Sample of Neutrinos}

The cascade sample likelihood test returned a test statistic value of zero, which sets a lower bound on the p-value of $49 \%$ and is consistent with no signal. The track event lies within the polar cap region $\delta<-85^{\circ}$ where scrambling in right ascension only does not provide independent background trials. However, as the event rate as a function of $\sin (\delta)$ is quite flat within this region, events with $\delta<-85^{\circ}$ were scrambled in both right ascension and declination. The test statistic returned by the likelihood analysis is zero, again consistent with no signal. The lower bound on the p-value is $71 \%$.

\section{Summary}

In this analysis, five years of data from IceTop and IceCube were used to search for $\mathrm{PeV}$ photons through maximum likelihood analyses: an all-sky scan, a correlation test with H.E.S.S. sources, and a correlation test with the IceCube 4-year HESE neutrino sample. For all cases, no evidence of significant signal was observed. The obtained sensitivity to point sources is the most stringent for $\mathrm{PeV}$ gamma rays yet reported. A search for a diffuse flux of $\mathrm{PeV}$ gamma rays correlated with the Galactic plane was also performed, which is covered in Ref. [15].

\section{References}

[1] IceCube Collaboration, M. Aartsen et al., Phys. Rev. D 13 (2013) 062002.

[2] H.E.S.S. Collaboration, F. Aharonian et al., ApJ 636 (2006) 777.

[3] The TeVCat catalog, http://tevcat.uchicago.edu.

[4] M. Ahlers and K. Murase, Phys. Rev. D90 (2014) 023010.

[5] N. Gupta, Astropart. Phys. 48 (2013) 75-77.

[6] R. J. Protheroe and P. L. Biermann, Astropart. Phys. 6 (1996) 45.

[7] IceCube Collaboration, M. Aartsen et al., JINST 12 (2017) 03012.

[8] IceCube Collaboration, R. Abbasi et al., NIM 700 (2013) 188.

[9] F. Halzen, A. Kappes, and A. Ó Murchadha, Phys. Rev. D 80 (2009) 083009.

[10] KASCADE Collaboration, D. Kang et al., J. Phys.: Conf. Ser. 632 (2015) 012013.

[11] Pedregosa et al., JMLR 12 (2011) 2825.

[12] CASA-MIA Collaboration, A. Borione et al., ApJ 493 (1998) 175.

[13] IceCube Collaboration, PoS ( ICRC2017) 514 (these proceedings).

[14] IceCube Collaboration, POS ( ICRC2015) 1098 (2016).

[15] IceCube Collaboration, POS ( ICRC2017) 705 (these proceedings).

[16] K. Gorski et al., Astrophys. J. 622 (2005) 759.

[17] H.E.S.S. Collaboration, F. Aharonian et al., A\&A 477 (2008) 353.

[18] S. Vernetto, and P. Lipari, EpJ 136 (2017) 03015.

[19] Guo et al., ApJ 835 (2017) 42. 